\title{
Energy Distribution in the Wind-Wave Spectrum
}

\author{
By \\ Kozo YoshidA \\ Geophysical Institute, Tokyo University, Tokyo \\ (Manuscript received 29 January, 1952)
}

\begin{abstract}
It has been suggested in a previously published paper that observed data of wave growth require for us to assume some sorts of energy transfer from shorter waves to longer waves in a wind-wave spectrum. In the present paper, we discuss such processes of energy transfer between component waves, by making use of the well known results which have been deduced semiempirically regarding the significant-wave. General features of wind-wave spectra for any stage of wave development being closely related to the process, the results from the present theory check with actual data of these spectra.
\end{abstract}

\section{Introduction.}

It has been suggested previously (Yoshida, 1950) by the author that energy transfer may occur from shorter waves to longer waves in a wave spectrum for any stage during the process of wave growth. Although the physical mechanism of such a transfer may not be well known, the limitation of the extreme steepness of individual waves and therefore the semi-continuous occurrence of breaking of shorter waves should be related to the process. The breaking of each component wave may occur with almost irregular interval, but occurs with longer interval than each own period and especially seems to do most frequently with period of the significant wave. Energy should then be transfired to longer waves, $r_{-}$sulting in special tendency to feed the significant wave.

The energy which is transfered from wind stresses my be distributed in any manner between each component wave, but it may be reasonable to assume that most part of such energy will enter into the significant wave. It might be said that, wind energy going to waves should be more widely distributed over the spectrum. In fact, the shorter waves may acquire more wind energy than the longer ones under the same conditions.

Therefore the longer waves than the significant wave will be supposed to receive only a small fraction of wind energy except for the waves which are very near in period to the significant wave. On the other hand, the shorter waves than the significant wave are those which have been in the earlier stages the significant waves, older at the stage considered and therefore the spectral energy in this range may not increase even if the energy enters. To simplify the matters, we shall confine ourselves to discussions only for the later stage than $\beta_{s}=0.4$ where $\beta_{s}$ denotes the wave age of the significant wave.

The present assumptions are made under above considerations. Discussions 
are made by dividing the whole spectrum into three parts: (1) the shorter wave range $\left(\beta<\beta_{s}\right)$, (2) the longer wave range $\left(\beta>\beta_{s}\right)$ and (3) the significant wave band. As time advances, the significant wave band shifts towards the longer period in the spectrum according to some empirical evidence and the significant-wave theories. Thus, the shorter wave range increases its width, while the longer wave region decreases in its wdth as time advances. To a first approximation, we assume that whole wind energy enters into the significant wave in each stage, and also assume that the energy thus entered passes on to the significant wave in the following stage. The total energy contained in the shorter wave region may be assumed not to change with time, because the wind en-rgy supplied to this system may be in the whole canceled by breaking process $s$ and consequently by turbulence. That contained in the longer wave region may not also change under the assumption as be stated above. It may be considered that the energy released during such turbulent processes from waves enters into the drift currents (1952, Yoshida).

It may be obvious, however, that the rate of en $\in$ rgy transfer under consideration cannot be determined without some additional conditions. In the present discussions, instead of going into any detailed mechanism of transfer, the determination may be made by comparing with the actual data. Hence the apparent transfer of energy presented in the results of this analysis might be interpreted in various ways. Although there may be obscurities in the present discussions because of the lack of data of wave spectra available for desired stages, the results may be fairly well consistent with the empirical data.

2.

The en rgy contained between $\beta$ and $\beta+d \beta$ may be given by

$$
E_{\beta} d \beta=\frac{\rho \pi^{2}}{2 g} W^{4} \delta_{\beta}^{2} \beta^{4} d \beta
$$

The d scussions may be made by dividing into three parts, of which the last part, the significant wave part, may be considered already given by SverdrupMunk's theory (1947). Furthermore, the rate of period increase of significant waves may be assumed in accordance with the significant wave theories.

In the present paper, use being made of the simpler formula obtained in the preceding paper by the author (Yoshida, 1951a), we have

$$
\beta_{s}(t)=\left(\frac{3}{2} A\right)^{\frac{1}{3}}\left(\frac{g t}{W}\right)^{\frac{1}{3}}
$$

Hence the energy equations for the first and the second part are given by

$$
\frac{d}{d t} \int_{0}^{\beta s} E_{\beta} d \beta=0
$$




$$
\frac{d}{d t} \int_{\beta_{s}}^{\infty} E_{\beta} d \beta=0
$$

respectively.

Using (1) and (2), (3) and (4) may be written as follows:

$$
\begin{array}{ll}
\int_{0}^{\beta_{s}} \frac{\partial \delta_{\beta}^{2}}{\partial t} \beta^{4} d \beta=-\left(\frac{g A}{2 W}\right) \delta_{s}^{2} \beta_{s}^{2} & \left(\beta<\beta_{s}\right) \\
\int_{\beta_{s}}^{\infty} \frac{\partial \delta_{\beta}^{2}}{\partial t} \beta^{4} d \beta=\frac{g A}{2 W} \delta_{s}^{2} \beta_{s}^{2} & \left(\beta>\beta_{s}\right)
\end{array}
$$

If particular solutions of (5) and (6) are assumed to have given by

and

$$
\begin{array}{ll}
\delta_{\boldsymbol{\beta}}^{2}=\delta_{i} \beta^{n_{i}} t^{m_{i}} & \left(\beta<\beta_{s}\right) \\
\delta_{\boldsymbol{\beta}}^{2}=\delta_{j} \beta^{n_{j}} t^{m_{j}} & \left(\beta>\beta_{s}\right)
\end{array}
$$

we have

$$
\begin{array}{ll}
\delta_{\beta_{s}}^{2}=\delta_{i i} \beta_{s}^{-5} & \left(\beta<\beta_{s}\right) \\
\delta_{s \beta^{2}}=\delta_{j j} \beta_{s}^{-5} & \left(\beta>\beta_{s}\right) \\
\delta_{\beta}^{2}=\delta_{s \beta^{2}}\left(\frac{t}{t_{s}}\right)^{m_{i}} & \left(\beta<\beta_{s}\right) \\
\delta_{\beta}^{2}=\delta_{s \beta^{2}}\left(\frac{t}{t_{s}}\right)^{m_{j}} & \left(\beta>\beta_{s}\right)
\end{array}
$$

If we assume the continuity of $E_{\beta}$ for $\beta=\beta_{s}$, we have $\delta_{i i}=\delta_{j j}=\delta_{0}$ (11) and (12) may be also written as follows:

$$
\begin{aligned}
& \delta_{\beta}^{2}=\delta_{0} \beta^{-5}\left(\frac{t}{t_{s}}\right)^{m_{i}}=\delta_{0} \beta^{-5}\left(\frac{\beta}{\beta_{s}}\right)^{-3 m_{i}} \\
& \delta_{\beta}^{2}=\delta_{0} \beta^{-5}\left(\frac{t}{t_{s}}\right)^{m_{j}}=\delta_{0} \beta^{-5}\left(\frac{\beta}{\beta_{s}}\right)^{-3 m_{j}}
\end{aligned}
$$

Derivation of these results, which are of physical significance, requires that $m_{\imath}<0$ and $m_{j}>0$. Further conditions that $d E_{\beta} / d \beta>0$ for $\beta<\beta_{s}$ and $d E_{\beta} / d \beta<0$ for $\beta>\beta_{s}$ requires that $m_{i}<-\frac{1}{3}$ and $m_{j}>-\frac{1}{3}$.

According to (13) we have

$$
E_{\beta} \propto \delta_{\beta}^{2} \beta^{4} \propto \beta^{-3} m_{i}-1
$$

which reduces to

$$
H_{T} \propto T^{-\frac{1}{2}\left(3 m_{i}+1\right)}
$$

It may be seen that such formulae may be valid $f \circ r m_{i}=-\frac{11}{3}$, when compared with the actual data of wave spectra which show $H(T) \propto T^{5}$. in every stage. For the region $\beta>\beta_{s}$, the actual data seem to indicate no definite relation as (15) and it may be difficult to determine the value of $m_{j}$. This may be ascribed to the co-existence of swell spectrum. 
These are illustrated in Fig. 1 and 2. Every point plotted in the figures is arranged from each wave spectrum, in order to check whether the ratio $(k)$ of $l_{n}\left|H_{T_{2}} / H_{T_{1}}\right|$ to $l_{n}\left|T_{2} / T_{1}\right|$ is constant for various s'ages and to determine the value of $m_{i}$.

Physical meanings of $m_{i}$ and $m_{j}$ may be understood by the following equations :

$$
\begin{aligned}
& \frac{\partial E_{\beta}}{\partial t}=\frac{m_{i}}{t} E_{\beta} \quad\left(\beta<\beta_{s}\right) ; \quad \frac{\partial E_{\beta}}{\partial t}=\frac{m_{j}}{t} E_{\beta}, \quad\left(\beta>\beta_{s}\right) \\
& \frac{\partial E_{\beta}}{\partial t}+\frac{m_{i}}{3 m_{i}+1} \frac{\beta}{t} \frac{\partial E_{\beta}}{\partial \beta}=0 \quad\left(\beta<\beta_{s}\right) ; \quad \frac{\partial E_{\beta}}{\partial t}+\frac{m_{j}}{3 m_{j}+1} \frac{\beta}{t} \frac{\partial E_{\beta}}{\partial \beta}=0 \quad\left(\beta>\beta_{s}\right)
\end{aligned}
$$

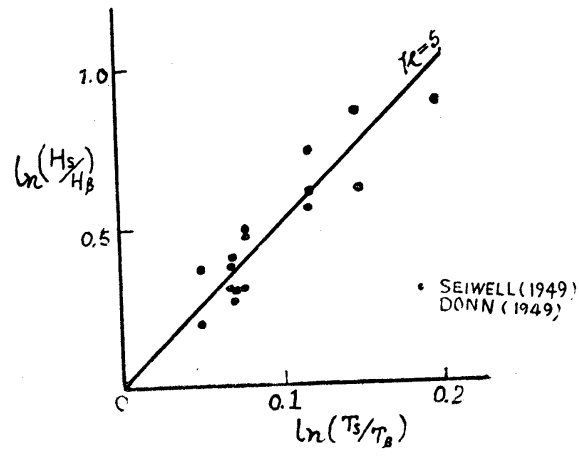

Fig. 1.

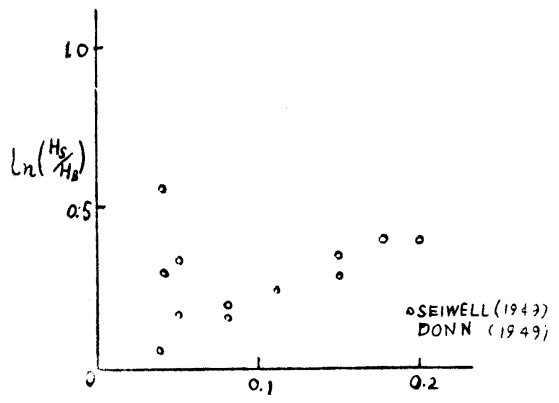

$\ln \left(T s / T_{\mathrm{s}}\right)$

Fig. 2.

\section{Connection with the "Significant-Wave Theory".}

The energy of the longer-end of the shorter wave range and of the shorter end of the longer wave range are equal to each other, and denoting it by $E_{s}$, we have

$$
\frac{d E_{s}}{d t}=\left.\frac{\partial E}{\partial t}\right|_{s}+\left.\frac{\partial E}{\partial \beta}\right|_{s} \frac{d \beta_{s}}{\bar{d} t}
$$

where $\partial E /\left.\partial t\right|_{s}$ and $\partial E /\left.\partial \beta\right|_{s}$ mean the time and pectral gradient respectively for $\dot{\beta}=\beta_{s}$.

From the present solutions for $\partial E / \partial t$ and $\partial E / \partial \beta$, we obtain the following equation:

$$
\frac{d E_{s}}{d t}=-\frac{1}{2} R_{T}\left(\beta_{s}\right)
$$

As was stated above, the wird energy may be assumed to enter into the significant wave band, at each moment this energy enter into the successively established significant wave at the moment and such entered energy may pass 
on to the significant wave in the subsequent stage. Under such assumptions, we may find the energy equation for significant wave band as the following:

$$
\frac{d E_{s}}{d t}=-\frac{1}{2} R_{T}\left(\beta_{s}\right)+R_{T}\left(\beta_{s}\right) \pm R_{N}\left(\beta_{s}\right)=\frac{1}{2} R_{T}(\beta) \pm R_{N}\left(\beta_{s}\right)
$$

This equation is similar as that obtained in Sverdrup-Munk's theory, and is just the same as that derived in the previous paper of the author (Yoshida, 1951). An interpretation of this equation is that, of wind energy entered into the significant wave, only $R_{H}$ may pass on to the subsequent significant wave and $R_{C}$ may be used to increase the significant wave period. Here $R_{H}$ and $R_{C}$ may be given by

$$
\begin{aligned}
& R_{H}=\frac{d E_{s}}{d t} \\
& R_{C}=\frac{E_{s}}{\beta_{s}} \frac{d \beta_{s}}{d t}=\frac{1}{2} R_{T}\left(\beta_{s}\right)
\end{aligned}
$$

The adjucent portions to the significant wave period may acquire no wind energy, while the wave velocity increases and energy may be used to increase the velocity. Thus the equations (17) and (18) may be well understood.

It may be remarked that the above discussions are based on a particular solution of the original equat ons. We can also obtain other types of solutions which indicate different manners of energy transfer. However, under the present situation, the above discussions may be considered in good consistence with our present understanding of the problems.

\section{Acknowledgement :}

The author wishes to express his sincere thanks to Prof. K. Hidaka for encouragement and support in carrying out this study.

\section{References}

Donn, W. L. (1949) : "Studies of waves and swell in the western North Atlantic". Trans. Amer. Geophys. Union, 30, p. 507-516.

Seiwell, H. R. (1949) : "The principles of time series analyses applied to ocean wave data". Proc. Nat. Acad. Sci., 35 (9), pp. 518-528.

Sverdrup, H. U. and W. H. Munk (1947): "Wind, sea and swell: Theory of relations for forecasting". U.S. Navy Hydrographic Office, Technical Report in Oceanography, No. 1, pp. 1-44. 
Yoshida, K. (1950): "On the mechanism of the period increase of waves in storm areas”. Geophys. Notes, 3 (24), p. 1-8.

Yoshida, K. (1951a) : "Theoretical derivation of equations for the Energy Split-up in the Wind waves". Geophys. Notes, 4 (6), p. 1-5.

Yoshida, K. (1952): "Dynamics on the co-existent system of waves and currents in the ocean. Part I." (unpublished). 